\title{
Direct detection of Dark Matter
}

\author{
P. Belli ${ }^{1,2}$ \\ ${ }^{1}$ Dip. di Fisica, Università di Roma "Tor Vergata", 00133 Rome, Italy \\ ${ }^{2}$ INFN, sez. Roma "Tor Vergata”, 00133 Rome, Italy
}

\begin{abstract}
An overview of the latest results of Dark Matter direct detection will be summarized, with particular care to the DAMA/LIBRA-phase1 results and the evidence with high confidence level obtained by exploiting the model independent Dark Matter annual modulation signature for the presence of Dark Matter particles in the galactic halo. Results from other experiments using different procedures, different techniques and different target-materials will be shortly discussed. Results, implications and experimental perspectives will be addressed.
\end{abstract}

\section{Introduction}

Nearly a century of experimental observations and theoretical arguments has pointed out that a large fraction of the Universe is composed by Dark Matter Particles and Dark Energy. Considering all the observational data coming from the study of the CMB, of the Supernovae Ia, of the Baryonic Acoustic Oscillations (BAO) and of the large-scale structures, the following contributions to $\Omega$ are obtained[1,2]: i) $\Omega_{r} \approx 5 \times 10^{-5}$ for the radiation density; ii) $\Omega_{b} \approx 0.05$ for the baryonic matter; iii) $\Omega_{d m} \approx 0.27$ for the non baryonic Dark Matter; iv) $\Omega_{\Lambda} \approx 0.68$ for the Dark Energy. A value $\Omega_{b} \approx 0.05$ for the baryonic matter is also supported by the Big-Bang nucleosynthesis (BBN), that is based on the predictions of the abundances of the light elements. This value is much larger than the cosmic density of the luminous matter, $\Omega_{\text {lum }} \approx 0.004$ [3], crediting that most baryons are dark and, probably, in the form of diffuse intergalactic medium [4].

The values of the cosmological parameters support that most of the matter in the Universe has a non baryonic nature.

As regards the light massive neutrino DM candidate, its density can be strongly constrained by cosmology; in fact, a value above the limit $\Omega_{v} \approx 0.03$ gives an unacceptable lack of small-scale structure $[5,6]$. In addition, a pure light massive neutrino scenario is ruled out by the measurements of the $\mathrm{CMB}$ radiation, which does not show sufficiently large inhomogeneity. Therefore, a significant role should be played by non-baryonic relic particles from the Big Bang, outside the Standard Model of particle physics; they have to be stable or with a lifetime comparable with the age of the Universe to survive up to now in a significant amount.

In theories extending the Standard Model of particle physics, many candidates as Dark Matter particles have been proposed having different nature and interaction types. Among the many DM candidates there are: SUSY particles (as e.g. neutralino or sneutrino in various scenarios), inelastic Dark Matter in various scenarios, electron interacting Dark Matter, a heavy neutrino of the 4-th family, sterile neutrino, Kaluza-Klein particles, selfinteracting Dark Matter, axion-like (light pseudoscalar and scalar candidate), mirror Dark Matter in various scenarios, Resonant Dark Matter, DM from exotic 4th generation quarks, 
Elementary Black holes, Planckian objects, Daemons, Composite DM, Light scalar WIMP through Higgs portal, Complex Scalar Dark Matter, specific two Higgs doublet models, exothermic DM, Secluded WIMPs, Asymmetric DM, Isospin-Violating Dark Matter, Singlet DM, Specific GU, SuperWIMPs, WIMPzilla, etc.. Moreover, even a suitable particle not yet foreseen by theories could be the solution or one of the solutions; in fact, considering the richness in particles of the visible matter which is less than $1 \%$ of the Universe density, one could also expect that the particle component of the Dark Matter in the Universe may be multicomponent.

Depending on the DM candidate, the interaction processes can be various, as e.g.: 1) elastic scatterings on target nuclei with either spin-independent or spin-dependent or mixed coupling; moreover, an additional electromagnetic contribution can arise, in case of few $\mathrm{GeV}$ candidates, from the excitation of bound electrons by the recoiling nuclei [7]; 2) inelastic scatterings on target nuclei with either spin-independent or spin-dependent or mixed coupling in various scenarios [8-13]; 3) interaction of light DM (LDM) either on electrons or on nuclei with production of a lighter particle [14]; 4) preferred interaction with electrons [15]; 5) conversion of DM particles into electromagnetic radiation [16]; 6) etc.. Often, the elastic scattering on target nuclei is the considered interaction process, but other processes are possible and considered in literature, as those aforementioned where also electromagnetic radiation is produced. Hence, considering the richness of particle possibilities and the existing uncertainties on related astrophysical (e.g. halo model and related parameters, etc.), nuclear (e.g. form factors, spin factors, scaling laws, etc.) and particle physics (e.g. particle nature and interaction types, etc.), a widely-sensitive model independent approach is mandatory. Most of the activities in the field are instead based on a particular a priori assumption on the nature of the DM particle and of its interaction, in order to try to overcome the limitation arising from their generally large originally measured counting rate.

The expected energy distribution for the interactions of Dark Matter particles in a terrestrial detector depends - among others - on their density and velocity distribution at Earth's position. However, the experimental observations regarding the dark halo of our Galaxy do not allow one to get information on this crucial aspect without introducing a model for the Galaxy matter density. Because of its simplicity, the isothermal sphere model (which consists in a spherical infinite system with a flat rotational curve) is a widely used assumption for the DM density distribution, and thus in the evaluation of Dark Matter expected rates. However many of its underlying assumptions (sphericity of the halo, absence of rotation, isotropy of the dispersion tensor, flatness of the rotational curve) are not strongly constrained by astrophysical observations. Moreover, the isothermal sphere is strictly unphysical and may only represent the behavior of the inner part of physical systems, since it has a total infinite mass and needs some cutoff at large radii. Thus, the use of more realistic halo models is mandatory in the interpretation and comparison procedures, since the model dependent results can significantly vary.

Generally, the halo models can be grouped in the following classes: i) spherically symmetric matter density with isotropic velocity dispersion; ii) spherically symmetric matter density with non-isotropic velocity dispersion; iii) axisymmetric models; iv) triaxial models; v) axisymmetric models either with a halo co-rotation or a halo counter-rotation. The possible rotation can be considered also for the other kinds of halo. A parameterization of these classes of halo models has been given in Ref. [17, 18], taking into account the available observational data; anyhow, information on the galactic dark halo can be obtained only in indirect way considering some hypotheses on its form and characteristic.

In conclusion, the uncertainties still present on the shape of the DM halo and on the density and velocity distribution prevent the definition of a "standard" halo and illustrate how 
the comparisons among the experiments of direct detection of DM particles (see later) can be consistent even just considering this particular aspect (also see Ref. [19]). Moreover, many other experimental and theoretical uncertainties exist and must be considered in a suitable comparison among the experiments of direct detection of DM particles.

\section{The Dark Matter particles detection}

It is worth noting that experiments at accelerators may prove - when they can state a solid model independent result - the existence of some possible DM candidate particles, but they could never credit by themselves that a certain particle is a/the only solution for Dark Matter particle(s). Moreover, DM candidate particles and scenarios (even e.g. in the case of the neutralino candidate) exist which cannot be investigated at accelerators. Thus, a model independent approach, a ultra-low-background suitable target material, a very large exposure and the full control of running conditions are mandatory to pursue a widely sensitive direct detection of DM particles in the galactic halo.

Considering the many available candidate particles and scenarios and the existing uncertainties on the astrophysical (e.g. halo model and related parameters, etc.), nuclear (e.g. form factors, spin factors, scaling laws, etc.) and particle physics (e.g. particle nature and interaction types, etc.), a widely-sensitive model independent approach is mandatory as well as a suitable exposure, and full control of the running condition over the whole data taking.

Indeed, most activities in the field release marginal exposures even after many years underground; they do not offer suitable information e.g. about operational stability and procedures during the running periods, and base their analysis on a particular a priori assumption on the nature of the DM particle and its interaction, and of all the used parameters.

In particular, the applied rejection and subtraction procedures to reduce the experimental counting rate, in order to derive a set of recoil-like candidates, is pursued by experiments as CDMS, EDELWEISS, CRESST, XENON, LUX, etc.. It is worth noting that the applied subtraction procedures are statistical and cannot offer an unambiguous identification of the presence of DM particle elastic scatterings because of the known existing recoil-like indistinguishable background; tails of the subtracted populations can play a role as well. Finally, the electromagnetic component of the counting rate, statistically "rejected" in this approach, can contain either the signal or part of it, and it will be lost. In the following few experimental activities are mentioned as examples.

In the double read-out bolometric technique, the heat signal and the ionization signal are used in order to discriminate between electromagnetic and recoil-like events. This technique is used by CDMS and EDELWEISS collaborations. In particular, the CDMS-II detector consists of $19 \mathrm{Ge}$ bolometers of about $230 \mathrm{~g}$ each one and of $11 \mathrm{Si}$ bolometers of about $100 \mathrm{~g}$ each one. The experiment released data for an exposure of about $194 \mathrm{~kg} \times$ day [20] using only $10 \mathrm{Ge}$ detectors in the analysis (discarding all the data collected with the other ones) and considering selected time periods for each detector. EDELWEISS employs a target fiducial mass of about $2 \mathrm{~kg}$ of Ge and has released data for an exposure of $384 \mathrm{~kg} \times$ day collected in two different periods (July-Nov 08 and April 09-May 10) [21] with a 17\% reduction of exposure due to run selection. These two experiments claim to have an "event by event" discrimination between noise + electromagnetic background and recoil + recoil-like (neutrons, end-range alphas, fission fragments,...) events by comparing the bolometer and the ionizing signals for each event. Thus, their results are, actually, largely based on huge data selections, as for example, the time cut analysis used to remove the so-called surface electrons that are distributed in both the electromagnetic and recoil bands. The stability, the unlinear response and the robustness of the reconstruction procedure are key points, as well as the 
associated systematical errors. In these experiments few recoil-like events survive the many selections/subtractions cuts applied in the data analysis; these events are generally interpreted in terms of background. As regards, in particular, their application to the search for time dependence of the data (such as the annual modulation signature), it would require - among other - to face the objective difficulty to control all the operating conditions - at the needed level $(<1 \%)$ - despite of the required periodical procedures e.g. for cooling and for radiation source introduction for calibration as well as of the limitation arising from the reachable duty cycle. For example, the attempt by CDMS-II to search for annual modulation in Ge target has been performed with a marginal exposure by using only 8 detectors over 30 and using - among others - data that are not continuous over the whole annual periods considered in the analysis [22]; the use of non-overlapping time periods, collected with detectors having different background rate within the signal box does not allow one to get any reliable result in the investigation of an effect at few percent level (see e.g. arguments in [23]).

Recently, the results of CDMS-II with the Si detectors were published in two close-intime data releases [24, 25]; while no events in six detectors (corresponding exposure of only $55.9 \mathrm{~kg} \times$ day before analysis cuts) were reported in the former [24], three events in eight detectors (corresponding raw exposure of $140.2 \mathrm{~kg} \times$ day) were reported over the residual background, estimated after subtraction: $\simeq 0.4$ in the second one [25]. The latter result could be interpreted - under certain assumptions - in terms of a DM candidate with spinindependent interaction and a mass around $10 \mathrm{GeV}$, which is compatible with interpretations of the model independent DM annual modulation result already reported by DAMA in terms of this kind of DM candidate and with some other hints reported by CRESST and CoGeNT (see later).

The XENON project uses instead dual phase liquid/gas detectors. Experiments exploiting such technique (as also LUX, DARKSIDE) perform statistical discrimination between nuclear recoil-like candidates and electromagnetic component of the measured counting rate through the ratio of the prompt scintillation signal $(S 1)$ and the delayed signal $(S 2)$ due to drifted electrons in the gaseous phase. The XENON100 experiment has released data for an exposure of 224.6 days, using a fiducial volume of just $34 \mathrm{~kg}$ of Xenon target mass [26]. See related discussions in literature about the detector response of such devices, in particular, to low energy recoils $[27,28]$. The technical performance of the apparata, confirmed also by similar experiments, has shown e.g. that: i) the detectors are affected by large non-uniformity; some kind of corrections may be estimated and applied, but significant systematics has to be accounted for; ii) the response of these detectors is not linear, i.e. the number of photoelectrons $/ \mathrm{keV}$ depends on the energy scale and depends also on the applied electric field; iii) the physical energy threshold is not suitably proved iv) the use of energy calibration lines from Xe activated by neutrons cannot be applied as routine and the studies on a possible calibration with internal sources in the same running conditions have not been realized so far; v) despite of the small light response (2.28 photoelectron/keVee), an energy threshold at 1.3 keVee is claimed; vi) the energy resolution is poor; vii) in the scale-up of the detectors the performances deteriorate; viii) the behaviour of the light yield for recoils at low energy is uncertain in every case. Recently, LUX reports the first results corresponding to an exposure of 85.3 days, using a fiducial volume of $118 \mathrm{~kg}$ [29]; similar considerations, as above, hold.

The CRESST experiment exploits the double read-out bolometric technique, using the heat signal due to an interacting particle in the $\mathrm{CaWO}_{4}$ crystals and the scintillation light produced. A statistical discrimination of nuclear recoil-like events from electromagnetic radiation is performed. As regards the cuts and selection procedures applied, most of the above discussion also holds. A previous run ( 8 detectors of $300 \mathrm{~g}$ each one, for an exposure of about $730 \mathrm{~kg} \times$ day [30]) showed, after selections, 67 nuclear recoil-like events have been 
observed in the Oxygen band. The background contribution estimated by authors ranges from 37 to 43 events, and does not account for all the observed events. The remaining number of events and their energy distribution could be interpreted - under certain assumptions - in terms of a DM candidate with spin-independent interaction and a mass in the range of $10-30 \mathrm{GeV}$. This result has been not confirmed in the last run [31], where a more marginal exposure has been used, confirming the difficulties to manage the systematics in such experiments.

Another positive hint for a signal of light Dark Matter candidates inducing just nuclear elastic scatterings have been reported by the CoGeNT experiment [32, 33]. The set-up is composed by a $440 \mathrm{~g}$, p-type point contact (PPC) Ge diode, with a very low energy threshold at 0.4 keVee. It is located in the Soudan Underground Laboratory. In the data analysis no discrimination between electromagnetic radiation and nuclear recoils is applied; only noise events are rejected. The experiment observes more events than they expect from an estimate of the background in the energy range 0.4-3.2 keVee. The energy spectrum of these events is compatible - under certain assumptions - with a signal produced by the interaction of a DM particle with a mass around $10 \mathrm{GeV}$. In addition, considering an exposure of $146 \mathrm{~kg} \times$ days CoGeNT experiment also reports an evidence at about 2.2 $\sigma$ C.L. of an annual modulation of the counting rate in (0.5-2) keVee with phase and period compatible - although the small confidence level - with a Dark Matter signal [33]. This result is compatible with interpretations of the DM model-independent annual modulation result already reported by DAMA in terms of this kind of DM candidate and with the possible hints reported above.

In conclusion, suitable experiments offering a model independent signature for the presence of Dark Matter particles in the galactic halo are mandatory.

\subsection{DM model independent signature and DAMA results}

To obtain a reliable signature for the presence of DM particles in the galactic halo, it is necessary to exploit a suitable model independent signature. With the present technology, one feasible and able to test a large range of cross sections and of DM particle halo densities, is the so-called DM annual modulation signature [34]. The annual modulation of the signal rate originates from the Earth revolution around the Sun. In fact, as a consequence of its annual revolution around the Sun, which is moving in the Galaxy traveling with respect to the Local Standard of Rest towards the star Vega near the constellation of Hercules, the Earth should be crossed by a larger flux of Dark Matter particles around $\sim 2$ June (when the Earth orbital velocity is summed to the one of the solar system with respect to the Galaxy) and by a smaller one around $\sim 2$ December (when the two velocities are subtracted). Thus, this signature has a different origin and peculiarities than the seasons on the Earth and than effects correlated with seasons (consider the expected value of the phase as well as the other requirements listed below). This DM annual modulation signature is very distinctive since the effect induced by DM particles must simultaneously satisfy all the following requirements: (1) the rate must contain a component modulated according to a cosine function; (2) with one year period; (3) with a phase that peaks roughly around $\sim 2$ nd June; (4) this modulation must be present only in a well-defined low energy range, where DM particles can induce signals; (5) it must be present only in those events where just a single detector, among all the available ones in the used set-up, actually "fires" (single-hit events), since the probability that DM particles experience multiple interactions is negligible; (6) the modulation amplitude in the region of maximal sensitivity has to be $\lesssim 7 \%$ in case of usually adopted halo distributions, but it may be significantly larger in case of some particular scenarios such as e.g. those in refs. [12, 35] 
At present status of technology it is the only DM model independent signature available in direct Dark Matter investigation that can be effectively exploited.

This signature has been exploited with large exposure - using highly radiopure $\mathrm{NaI}(\mathrm{Tl})$ as target material - by the former DAMA/NaI ( $\simeq 100 \mathrm{~kg}$ sensitive mass) experiment and the present DAMA/LIBRA ( $\simeq 250 \mathrm{~kg}$ sensitive mass), within the DAMA project [14-16, 18, 36-55].

In particular, the experimental observable in DAMA experiments is the modulated component of the signal in $\mathrm{NaI}(\mathrm{Tl})$ target and not the constant part of it, as done in the other approaches aforementioned.

The whole DAMA/LIBRA-phase1 results correspond to seven annual cycles for an exposure of 1.04 ton $\times y r$ [44-46]. Considering these data together with those previously collected by DAMA/NaI over 7 annual cycles $(0.29$ ton $\times y r)$, the total exposure collected over 14 annual cycles is 1.33 ton $\times y r$; this is orders of magnitude larger than the exposures typically collected in the field.

The DAMA/NaI set up and its performances are described in [18, 37-39], while the DAMA/LIBRA set-up and its performances are described in Ref. [43, 45]. In particular, the sensitive part of the DAMA/LIBRA set-up is made of 25 highly radiopure $\mathrm{NaI}(\mathrm{Tl})$ crystal scintillators placed in a 5-rows by 5-columns matrix; each crystal is coupled to two low background photomultipliers working in coincidence at single photoelectron level. For details see Ref. [36, 43].

The DAMA/LIBRA-phase1 results give evidence for the presence of Dark Matter particles in the galactic halo, on the basis of the exploited model independent DM annual modulation signature by using highly radio-pure $\mathrm{NaI}(\mathrm{Tl})$ target, at $7.5 \sigma$ C.L. Including also the first generation DAMA/NaI experiment (cumulative exposure 1.33 ton $\times \mathrm{yr}$, corresponding to 14 annual cycles), the C.L. is $9.3 \sigma$ and the modulation amplitude of the single-hit events in the (2-6) $\mathrm{keV}$ energy interval in $\mathrm{NaI}(\mathrm{Tl})$ target is: $(0.0112 \pm$ $0.0012) \mathrm{cpd} / \mathrm{kg} / \mathrm{keV}$; the measured phase is $(144 \pm 7)$ days and the measured period is $(0.998 \pm 0.002) \mathrm{yr}$, values well in agreement with those expected for DM particles. No systematic or side reaction able to mimic the exploited DM signature has been found or suggested by anyone over more than a decade.

After a first upgrade in 2008, a further upgrade of DAMA/LIBRA has been performed at the end of 2010 when all the PMTs have been replaced with new ones having higher quantum efficiency [48]. Since then, after a test and optimization period, the DAMA/LIBRA-phase2 is continuously running in order: (1) to increase the experimental sensitivity lowering the software energy threshold of the experiment; (2) to improve the corollary investigation on the nature of the DM particle and related astrophysical, nuclear and particle physics arguments; (3) to investigate other signal features and second order effects. DAMA/LIBRA also continue its study on several other rare processes [51-55].

\subsubsection{Implications and comparisons}

The DM model independent evidence obtained by DAMA is compatible with a wide set of scenarios regarding the nature of the DM candidate and related astrophysical, nuclear and particle Physics. For example some given scenarios and parameters are discussed e.g. in refs. [14-16, 18, 39-42, 44, 50]. Further large literature is available on the topics [56]; other possibilities are open.

It is worth noting that no other experiment exists, whose result can be directly compared in a model-independent way with those by DAMA/NaI and DAMA/LIBRA. Some activities (e.g. [20, 21, 26]) claim a model-dependent exclusion under many largely 
arbitrary assumptions (see for example discussions in Refs. [18, 27, 28, 39, 44]). However, often some critical points exist in their experimental aspects, as mentioned above, and the existing experimental and theoretical uncertainties are generally not considered in their presented single model dependent result; moreover, implications of the DAMA results are often presented in incorrect/partial/unupdated way. Both the accounting of the existing uncertainties and the existence of alternative scenarios (see literature) allow one to note that model dependent reults by indirect and direct experiments actually are not in conflict with the DAMA model independent result.

\section{Prospects for the DM directionality approach}

The directionality approach - based on the study of the correlation between the recoil direction of the target nuclei and the Earth motion in the galactic rest frame - can offer a good approach to study those DM candidate particles able to induce just nuclear recoils. In fact, the dynamics of the rotation of the Milky Way galactic disc through the halo of DM causes the Earth to experience a wind of DM particles apparently flowing along a direction opposite to that of the solar motion relative to the DM halo. Hence, in the case of DM candidate particles interacting with nuclei the induced nuclear recoils are expected to be strongly correlated with the impinging direction of DM, while the background events are not; therefore, the study of the nuclear recoils direction can offer a way for pointing out the presence of these DM candidate particles.

In the practice, this approach has some technical difficulties because it is arduous to detect the short recoil track. Different techniques are under consideration but, up to now, they are at R\&D stage and have not produced yet competitive results in the field (see e.g. the DRIFT project or the DM-TPC experiment). In fact, they are generally limited by the difficulty of detecting very short tracks and of achieving high stability, large sensitive volume and very good spatial resolution.

To overcome such a difficulty, it has been suggested the use of anisotropic scintillator detectors [57-59]; their use was proposed for the first time in [57] and revisited in [58]. In particular, low background $\mathrm{ZnWO}_{4}$ crystal scintillators have been recently proposed since their features and performances are very promising [60]. In fact, both the light output and the scintillation pulse shape depend on the impinging direction of heavy particles (p, alpha, nuclear recoils, etc.) with respect to the crystal axes and can supply two independent ways to study the directionality and to discriminate the electromagnetic background (that does not give rise to any anisotropic effects).

Other advantages offered by $\mathrm{ZnWO}_{4}$ detectors are very good radio-purity levels (about $0.1 \mathrm{cpd} / \mathrm{kg} / \mathrm{keV}$ at low energy) and the possibility to reach energy thresholds at $\mathrm{keV}$ level. Both these features can also be improved (e.g., the light yield shows a significant enhancement when working at low temperatures - about $100 \mathrm{~K}$ - and better radiopurity levels can be reached with dedicated R\&D). A detailed discussion can be found in [60].

It is also worth to mention the new idea of using DNA for nanometer tracking [61].

\section{Conclusions and perspectives}

Large efforts are in progress with different approaches and target materials to investigate various kinds of DM candidates and scenarios. Due to the difficulty of measuring at very low energy, several techniques still would require further work for results' qualifications before enlarging their target mass.

As regards the possibility to exploit the directionality for some DM candidates, new efforts have to be encouraged towards a first realistic exploitation. 
The DM model independent annual modulation signature with widely sensitive target materials still remains a major approach, offering an unique possibility for detection; it requires well known techniques, full proved detector stability, well known and proved detector response in all the aspects, etc. At present the DAMA positive model independent evidence for the presence of DM particles in the galactic halo is supported at $9.3 \sigma$ C.L. (on a cumulative exposure of 1.33 ton $\times y r$ i.e. 14 annual cycles of DAMA/NaI and DAMA/LIBRA). It has been shown in literature that this is compatible with many DM scenarios.

I have been also recalled the recent possible positive hints exploiting different approaches and different target materials, and the existing uncertainties in the model dependent results and comparisons.

Finally very useful complementary results can arise from experiment exploiting other target detectors and approaches adopting adequately safe experimental procedures. For completeness, it is also worth to mention the efforts to investigate the axion candidate and in particular the present efforts of the ADMX experiment.

\section{References}

[1] J. Beringer et al., Phys. Rev. D 86 (2012) 010001

[2] Planck coll., arXiv:1303.5076v1

[3] M. Fukugita and P.J.E. Peebles, Astrophys. J. 616 (2004) 643

[4] R. Cen and J.P. Ostriker, Astrophys. J. 514 (1999) 1

[5] R. A. C. Croft et al., Phys. Rev. Lett. 83 (1999) 1092

[6] O. Elgaroy et al., astroph/0204152

[7] R. Bernabei et al., Int. J. Mod. Phys. A 22 (2007) 3155

[8] R. Bernabei et al., Eur. Phys. J. C 23 (2002) 61

[9] S. Chang et al., Phys. Rev. D 79 (2009) 043513

[10] S. Chang et al., Phys. Rev. Lett. 106 (2011) 011301

[11] S. Chang et al., J. Cosm. Astrop. Phys. 08 (2010) 018

[12] D. Tucker-Smith and N. Weiner, Phys. Rev. D 64 (2001) 043502

[13] D. Tucker-Smith and N. Weiner, Phys. Rev. D 72 (2005) 063509

[14] R. Bernabei et al., Mod. Phys. Lett. A 23 (2008) 2125

[15] R. Bernabei et al., Phys. Rev. D 77 (2008) 023506

[16] R. Bernabei et al., Int. J. Mod. Phys. A 21 (2006) 1445

[17] P. Belli et al., Phys. Rev. D 66 (2002) 043503

[18] R. Bernabei el al., La Rivista del Nuovo Cimento 26(1) (2003) 1-73

[19] Y. Y. Mao, L. E. Strigari and R. H. Wechsler, arXiv:1304.6401

[20] Z. Ahmed et al., Science 327 (2010) 1619

[21] E. Armengaud et al., Phys. Lett. B 702 (2011) 329

[22] Z. Ahmed et al., arxiv:1203.1309

[23] J.I. Collar and N.E. Fields, arxiv:1204.3559

[24] R. Agnese et al., arXiv:1304.3706

[25] R. Agnese et al., arXiv:1304.4279

[26] E. Aprile et al., Phys. Rev. Lett. 107 (2011) 131302

[27] R. Bernabei et al., "Liquid Noble gases for Dark Matter searches: a synoptic survey", Exorma Ed., Roma, ISBN 978-88-95688-12-1, 2009, pp. 1-53, arXiv:0806.0011v2

[28] J.I. Collar and D.N. McKinsey, arXiv:1005.0838; arXiv:1005.3723; J.I. Collar,

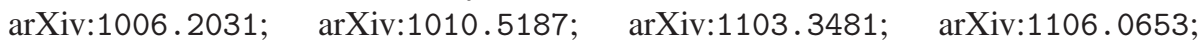
arXiv:1106.3559 
[29] D.S. Akerib et al., Phys. Rev. Lett. 112 (2014) 091303

[30] G. Angloher et al., Eur. Phys. J. C 72 (2012) 1971

[31] G. Angloher et al., arXiv:1407.3146

[32] C.E. Aalseth et al., Phys. Rev. Lett. 106 (2011) 131301

[33] C.E. Aalseth et al., arXiv: 1401.3295

[34] A.K. Drukier, K. Freese, and D.N. Spergel, Phys. Rev. D 33 (1986) 3495; K. Freese, J. A. Frieman and A. Gould, Phys. Rev. D 37 (1988) 3388

[35] K. Freese et al., Phys. Rev. D 71 (2005) 043516; K. Freese et al., Phys. Rev. Lett. 92 (2004) 11301

[36] R. Cerulli, at this Conference

[37] R. Bernabei et al., Il Nuovo Cim. A 112 (1999) 545

[38] R. Bernabei et al., Eur. Phys. J. C 18 (2000) 283

[39] R. Bernabei et al., Int. J. Mod. Phys. D 13 (2004) 2127

[40] R. Bernabei et al., Eur. Phys. J. C 47 (2006) 263

[41] R. Bernabei et al., Int. J. Mod. Phys. A 22 (2007) 3155

[42] R. Bernabei et al., Eur. Phys. J. C 53 (2008) 205

[43] R. Bernabei et al., Nucl. Instr. and Meth. A 592 (2008) 297

[44] R. Bernabei et al., Eur. Phys. J. C 56 (2008) 333

[45] R. Bernabei et al., Eur. Phys. J. C 67 (2010) 39

[46] R. Bernabei et al., Eur. Phys. J. C 73 (2013) 2648

[47] P. Belli et al., Phys. Rev. D 84 (2011) 055014

[48] R. Bernabei et al., J. of Instr. 7 (2012) P03009

[49] R. Bernabei et al., Eur. Phys. J. C 72 (2012) 2064

[50] R. Bernabei et al., Int. J. of Mod. Phys. A 28 (2013) 1330022

[51] R. Bernabei et al., Eur. Phys. J. C 62 (2009) 327

[52] R. Bernabei et al., Eur. Phys. J. C 72 (2012) 1920

[53] R. Bernabei et al., Eur. Phys. J. A 49 (2013) 64

[54] R. Bernabei et al., Eur. Phys. J. C 74 (2014) 2827

[55] R. Bernabei et al., Eur. Phys. J. C 74 (2014) 3196

[56] A. Bottino et al., Phys. Rev. D 81 (2010) 107302; N. Fornengo et al., Phys. Rev. D 83 (2011) 015001; A.L. Fitzpatrick et al., Phys. Rev. D 81 (2010) 115005; D. Hooper et al., Phys. Rev. D 82 (2010) 123509; A.V. Belikov et al., Phys. Lett. B 705 (2011) 82; E. Kuflik et al., Phys. Rev. D 81 (2010) 111701; S. Chang et al., Phys. Rev. D 79 (2009) 043513; S. Chang et al., Phys. Rev. Lett. 106 (2011) 011301; R. Foot, Phys. Rev. D 81 (2010) 087302; Y. Bai, P.J. Fox, JHEP 0911 (2009) 052; J. Alwall et al., Phys. Rev. D 81 (2010) 114027; M.Yu. Khlopov et al., arXiv:1003.1144; S. Andreas et al., Phys. Rev. D 82 (2010) 043522; J. Kopp et al., JCAP 1002 (2010) 014; V. Barger et al., Phys. Rev. D 82 (2010) 035019; P. Belli et al., Phys. Rev. D 84 (2011) 055014; J. L. Feng et al., Phys. Lett. B 703 (2011) 124; A. Bottino et al., Phys. Rev. D 85 (2012) 095013; C.E. Aalseth et al., arXiv:1401.3295; R. Foot, arXiv:1401.3965; Q. Wallemacq, arXiv:1401.5243; S. Scopel et al., arXiv:1405.0364; C. Arina et al., arXiv:1406.5542; R. Foot, arXiv:1407.4213; G. Barello et al., arXiv:1409.0536; Q. Wallemacq, arXiv: 1411.3178

[57] P. Belli et al., Il Nuovo Cim. C 15 (1992) 475

[58] R. Bernabei et al., Eur. Phys. J. C 28 (2003) 203

[59] N.J.C. Spooner et al., IDM Workshop 481 (1997); Y. Shimizu et al., Nucl. Instrum. and Meth. A 496 (2003) 347

[60] F. Cappella et al., Eur. Phys. J. C 73 (2013) 2276

[61] A. Drukier et al., arXiv:1206.6809 